\title{
Unveiling the Direct Correlation between the CVD-Grown Graphene and the Growth Template
}

\author{
Junghyun Lee $\mathbb{D},{ }^{1}$ Jihyung Seo $\mathbb{D},{ }^{1}$ Sungchul Jung, ${ }^{2}$ Kibog Park $\mathbb{D},^{2}$ and Hyesung Park $\mathbb{D}^{1}$ \\ ${ }^{1}$ Department of Energy Engineering, School of Energy and Chemical Engineering, Low Dimensional Carbon Materials Center, \\ Ulsan National Institute of Science and Technology (UNIST), Ulsan 44919, Republic of Korea \\ ${ }^{2}$ Department of Physics, Ulsan National Institute of Science and Technology (UNIST), Ulsan 44919, Republic of Korea
}

Correspondence should be addressed to Hyesung Park; hspark@unist.ac.kr

Received 29 April 2018; Accepted 24 June 2018; Published 19 August 2018

Academic Editor: Mohamed Bououdina

Copyright ( 2018 Junghyun Lee et al. This is an open access article distributed under the Creative Commons Attribution License, which permits unrestricted use, distribution, and reproduction in any medium, provided the original work is properly cited.

Chemical vapor deposition (CVD) is known to produce continuous, large-area graphene sheet with decent physical properties. In the CVD process, catalytic metal substrates are typically used as the growth template, and copper has been adopted as the representative material platform due to its low carbon solubility and resulting monolayer graphene growth capability. For the widespread industrial applications of graphene, achieving the high-quality is essential. Several factors affect the qualities of CVD-grown graphene, such as pressure, temperature, carbon precursors, or growth template. In this work, we provide detailed analysis on the direct relation between the metallic growth substrate (copper) and overall properties of the resulting CVD-grown graphene. The surface morphology of copper substrate was modulated via simple chemical treatments, and its effect on physical, optical, and electrical properties of graphene was analyzed. Based on these results, we propose a simple synthesis route to produce high-quality, continuous, monolayer graphene sheet, which can facilitate the commercialization of CVD graphene into reality.

\section{Introduction}

Graphene is one-atomic-layer-thick carbon atoms with honeycomb lattice structures, which is the first kind of 2-dimensional materials that have been experimentally discovered since 2004 [1]. With its outstanding physical and chemical properties, graphene has been proposed as an emerging nanomaterial for the next-generation electronic devices, such as flexible electronics, high-frequency transistors, or photonic devices, exceeding the performance of conventional devices [2-9]. Ever since its experimental demonstrations, various synthesis routes have been introduced for the production of graphene [10-16]. To list a few, mechanical exfoliation can yield high-quality of pristine graphene, but it lacks the large-scale production capability, an essential feature required for industrial applications [1]. Liquid-phase exfoliation technique, commonly used in the synthesis of graphene oxide (GO) or graphene intercalation compound (GIC), is promising for large-scale production. However, this approach suffers from the relatively low- quality of graphene as well as the difficulty in layer control, thereby limiting its applications in high-performance electronic devices [12-14]. Chemical vapor deposition (CVD) method, which utilizes catalytic metal substrates as the growth template, is well-known to produce large-area, highquality graphene films suitable for electronic applications $[17,18]$. In CVD process, several factors can affect the properties of the grown graphene film. In particular, the electrical properties of graphene are highly dependent on the grain boundaries, which induce charge carrier scatterings and thus deteriorate the carrier mobility [19]. Since the grain boundaries mostly originate from the lattice mismatch during the growth process that is primarily influenced by the orientation of catalytic metal substrates, single-crystal metals like Ge (110), $\mathrm{Cu}$ (111), or $\mathrm{Ni}$ (111) have been investigated as the alternative growth template to avoid such grain boundaries, and high-quality single-crystalline graphene has been demonstrated which epitaxially grew onto those substrates [20-22]. However, the process involved with the production of single-crystalline materials is generally cost-intensive, 


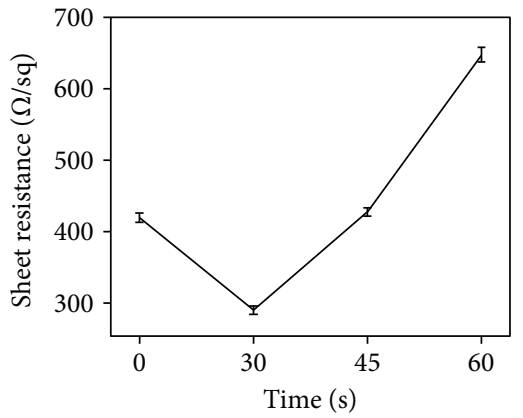

(a)

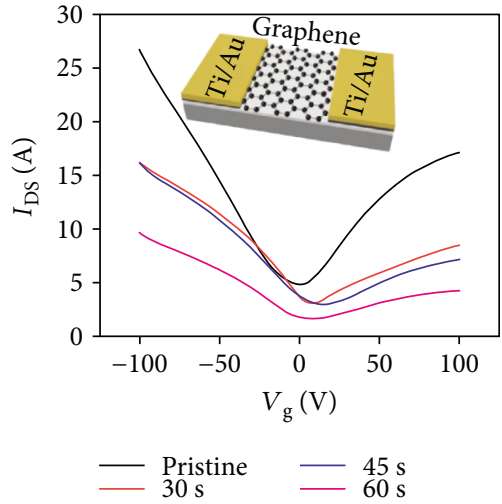

(b)

FIGURE 1: Electrical properties of the graphene film showing the (a) sheet resistance and (b) FET mobility characteristics (inset image indicates the FET device structure).

which is not suitable for widespread industrial applications. Another strategy to improve the electrical properties of graphene is to increase the domain size, which can be achieved by suppressing the nucleation densities during the synthesis [23-25]. Such approaches include introducing oxygen gas, using enclosed metal catalysts, or resolidifying metallic substrates to create smooth surfaces during the growth stage. Among these various approaches in the CVD process, modifying the surface morphologies of catalytic growth substrates is considered one of the most viable approaches for obtaining high-quality graphene. Mechanical or chemical treatments are typically used in such approach which significantly reduces the impurities present in the metal catalysts, the major source of the nucleation center, leading to the improved carrier mobility [26-32]. In this work, we revisit the role of surface morphology of metal catalyst to the quality of CVD graphene using commercially available copper foil via simple chemical treatments and analyze the resulting physical properties. Chemical treatments on the growth substrates typically involve etching process, and we show that proper control of the metal catalyst pretreatment can be a simple yet facile approach to enhance the overall quality of CVD-grown graphene.

\section{Experimental}

2.1. Synthesis and Transfer of Graphene. Copper foil (99.8\%, $25 \mu \mathrm{m}$ thickness, 13,382, Alfa Aesar) was pretreated with $5.8 \%$ nitric acid $\left(\mathrm{HNO}_{3}\right)$ under varying exposure time from $0 \mathrm{~s}$ (reference sample) to $60 \mathrm{~s}$ and washed with D.I. water, followed by acetone and isopropyl alcohol (IPA). We note that the synthesis result of reference sample exhibited relatively low reproducibility compared with those samples synthesized from the pretreated metal catalysts, likely due to the impurities present on the metal catalyst surface. After the chemical treatment, the copper foil turned to slightly dark brown due to the oxidation. The copper foil was then loaded into the CVD chamber which was purged with $\operatorname{Ar}(100 \mathrm{sccm})$ for $10 \mathrm{~min}$. After annealing the copper under $\mathrm{H}_{2}(10 \mathrm{sccm})$ environment for $30 \mathrm{~min}$ at $1000^{\circ} \mathrm{C}$, graphene synthesis was carried out with $\mathrm{CH}_{4}(20 \mathrm{sccm})$ and $\mathrm{H}_{2}(10 \mathrm{sccm})$ for
30 min ( 30 mTorr). After the growth, the samples were rapidly cooled down to room temperature.

The as-grown graphene was transferred to $\mathrm{SiO}_{2}$ $(300 \mathrm{~nm}) / \mathrm{Si}$ substrate via PMMA- (poly(methyl methacrylate)-) assisted transfer method. In brief, PMMA (9\% in anisole) was spin-coated onto the graphene, which was dried in oven to remove the solvent. Copper foil was then etched away by ferric chloride solution, and the PMMA/ graphene stack was thoroughly rinsed with mild hydrochloric acid and D.I. water. After transferring to the target substrate, PMMA was finally removed by acetone.

2.2. Characterization. Graphene channel was formed by standard photolithography and $\mathrm{O}_{2}$ plasma etching process. Source and drain contacts $(\mathrm{Ti} / \mathrm{Au}, 10 / 50 \mathrm{~nm}$ ) were deposited by e-beam evaporation system. Heavily $p$-doped $\mathrm{Si}$ substrate was used for the gate electrode, and the $\mathrm{SiO}_{2}$ layer was working as the gate insulator. Source-drain voltage was fixed to $0.05 \mathrm{~V}$, and gate voltage $\left(V_{\mathrm{G}}\right)$ sweep was from $-100 \mathrm{~V}$ to $100 \mathrm{~V}$ to measure the source-drain currents $\left(I_{\mathrm{DS}}\right)$. The obtained $I_{\mathrm{DS}}-V_{\mathrm{G}}$ curve is shown in Figure 1(b). Sheet resistance of graphene was measured by 4-point probe (CMT-SR2000N, Advanced Instrument Technology). Surface morphology of graphene was analyzed by optical microscopy (OM, Eclipse LV150, Nikon) and scanning electron microscopy (SEM, S-4800, Hitachi). Raman spectra were obtained from Alpha300R, WITec, with excitation wavelength at $532 \mathrm{~nm}$. Transmittance was measured by UV-vis NIR spectroscopy (Carry 5000, Agilent).

\section{Results and Discussion}

In this work, low-pressure CVD with copper substrate was used for the graphene synthesis and nitric acid was applied to modify the surface morphology of the as-received copper foil (see Experimental). In general, $\mathrm{HNO}_{3}$ reacts with copper and forms $\mathrm{Cu}\left(\mathrm{NO}_{3}\right)_{2}$ which is soluble in water as described in the following chemical reaction [26].

$$
\mathrm{Cu}(\mathrm{s})+4 \mathrm{HNO}_{3}(\mathrm{aq}) \rightarrow \mathrm{Cu}\left(\mathrm{NO}_{3}\right)_{2}(\mathrm{aq})+2 \mathrm{NO}_{2}(\mathrm{~g})+2 \mathrm{H}_{2} \mathrm{O}
$$




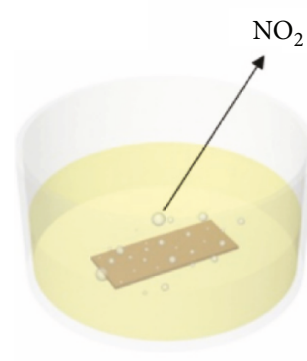

Nitric acid

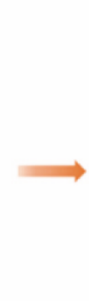

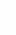

(nitric acid

-

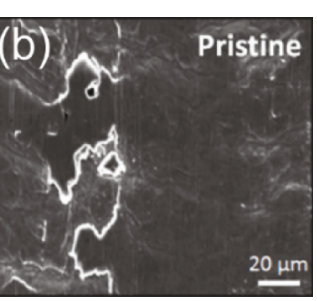

(b)

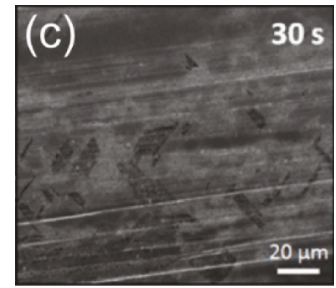

(c)

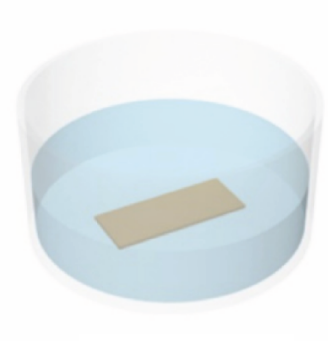

D.I. water

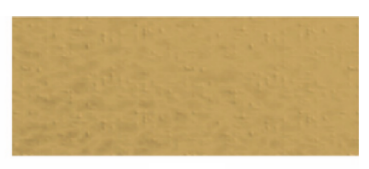

$30 \mathrm{~s}$

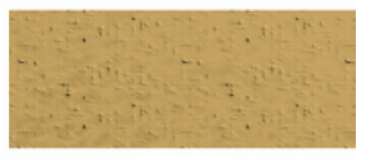

$45 \mathrm{~s}$

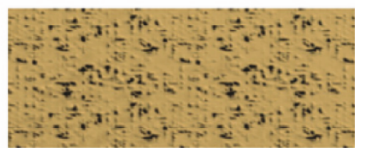

$60 \mathrm{~s}$

(a)

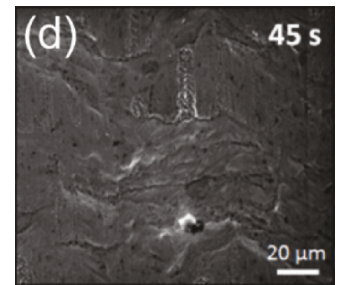

(d)

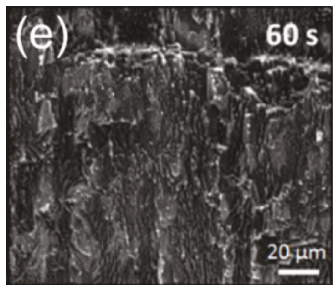

(e)

Figure 2: (a) Schematic of copper pretreatment process via nitric acid. The surface roughness of copper increases with longer acid treatment time. SEM images of surface morphologies of (b) pristine copper and (c-e) after various acid treatment conditions.

The overall reaction is dependent on the nitric acid treatment time, where the amount of $\mathrm{Cu}\left(\mathrm{NO}_{3}\right)_{2}$ increases with prolonged treatments which renders the copper surface morphology more rough and nonuniform as illustrated in Figure 2(a), leading to potential defect sites during the CVD process. The as-received pristine copper, with only conventional solvent rinsing such as acetone or isopropanol, typically exhibits bright regions due to the impurities naturally present in the copper (Figure 2(b)), which can be eliminated with such acid treatment. As shown in Figure 2(c), these bright spots are substantially reduced, and the surface becomes relatively smoother only after $30 \mathrm{~s}$ of acid treatment. However, extended chemical treatments typically result in highly nonuniform surface morphology due to the continued etching of the copper (Figure 2(d)). After $60 \mathrm{~s}$ of such reaction, the resulting morphology of copper becomes significantly rough compared to the pristine one despite the removal of impurities as shown in Figure 2(e).

To verify the effect of copper morphology to the growth of CVD graphene, surface morphologies of the as-transferred graphene grown on copper substrates with various pretreatment conditions were analyzed. Figure 3 shows the $\mathrm{OM}$ and SEM images of graphene sheets prepared under the same synthesis conditions and transferred onto the $\mathrm{SiO}_{2}$ substrates. Several multilayer graphene domains are observed from the pristine copper substrate due to the large number of impurities which act as the nucleation center during the growth stage (red arrow, Figure 3(a)) [33]. The presence of typical wrinkle formation is also observed which originates from the difference in thermal expansion coefficients between the graphene and metal substrate as well as during the transfer process (blue arrow, Figure 3(b)) [34]. The bright spot is the polymer residue resulting from the transfer process (white arrow, Figure 3(b)) [35]. Such formation of multilayer graphene was significantly suppressed with copper substrate treated by $30 \mathrm{~s}$ of nitric acid as shown in Figures 3(b) and 3(f). However, multilayer graphene started to reappear with increasing acid treatment to $45 \mathrm{~s}$ due to the corresponding increase in roughness of the copper substrate (Figures 3(c) and 3(g)). Activated carbon atoms tend to aggregate more at the rough surface, which consequently increases the probability of multilayer formation $[11,25]$. After $60 \mathrm{~s}$ treatment time, the presence of multilayer graphene even became comparable to that of the pristine copper case as shown in Figures 3(d) and 3(h). Figure 3(i) illustrates the distribution of monolayer graphene formation over the multilayer of the above, showing that monolayer graphene is mostly synthesized for the $30 \mathrm{~s}$ treatment case.

Optical transmittance of the respective graphene films was measured to evaluate the appearance of multilayer graphene (Supporting Information, Figure S1). Transmittance of $30 \mathrm{~s}$ sample was $\sim 97.5 \%$ at $550 \mathrm{~nm}$, similar to the theoretical value of monolayer graphene film (97.7\%) [7]. This value was decreased to $\sim 97.2$ and $~ 96.5 \%$ with increasing acid treatments of 45 and 60 s, respectively, whereas the pristine copper sample showed $\sim 96.9 \%$ transmittance likely due to the presence of multilayer graphene and light scattering induced from the rough graphene surface lowering the transmittance. These optical microscopy and transmittance results confirm that the 


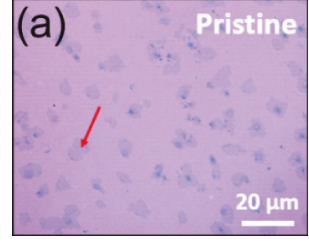

(a)

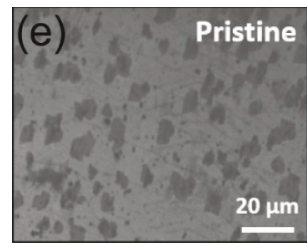

(e)

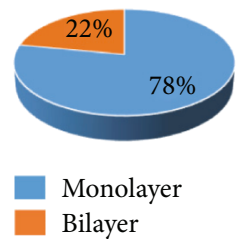

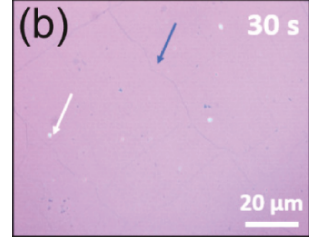

(b)

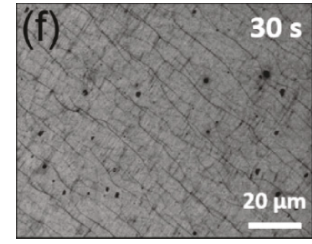

(f)
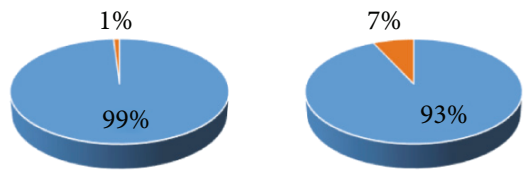

(g)

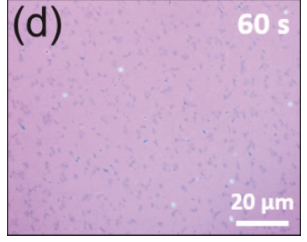

(d)
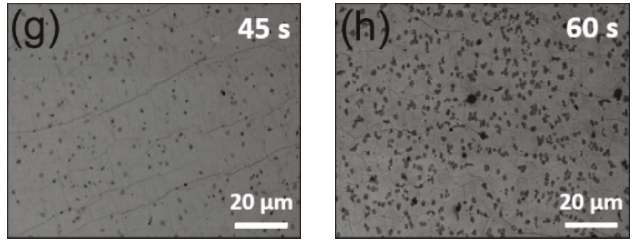

(h)

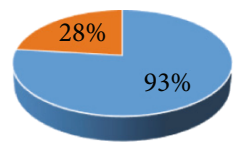

(i)

Figure 3: Optical (a-d) and SEM (e-h) images of graphene synthesized from various types of copper substrates and transferred onto the $\mathrm{SiO}_{2}$ substrates and (i) the corresponding distribution on the ratio of monolayer to bilayer graphene formation. Red, blue, and white arrows indicate few layer graphene, wrinkles, and PMMA residues on the graphene, respectively.

formation of multilayer graphene is highly affected by the morphology of underlying catalytic growth substrate, which can be modulated and remedied via appropriate pretreatment conditions.

Next, Raman spectroscopy analysis was performed to investigate the overall quality of graphene sheets prepared from different types of copper substrates. All graphene films under study exhibit primarily monolayer characteristics with $G$ and $2 D$ peaks at $\sim 1580$ and $2680 \mathrm{~cm}^{-1}$, respectively, and intensity ratio $I(2 \mathrm{D}) / I(\mathrm{G})$ of nearly 2 as shown in Figure 4(a) [20]. We note that the intensity of D peak at $\sim 1350 \mathrm{~cm}^{-1}$, representing the degree of defects in $\mathrm{sp}^{2}$ carbon domains, increases with acid treatment time, that is, the roughness of metal substrates (Figure 4(b)). We speculate that the increase of defect is related to multilayer graphene patches, which is formed by surface impurities or rough surfaces [36]. The value of full width at half maximum (FWHM) of 2D peak is another indicator commonly used to evaluate the quality of graphene, where the sharp peak is associated with the high-quality [37]. FWHM of 2D peak from $30 \mathrm{~s}$ sample is $\sim 35 \mathrm{~cm}^{-1}$ which is comparable to that of the mechanically exfoliated graphene, and gradually increases to $\sim 43$ and $\sim 52 \mathrm{~cm}^{-1}$ for 45 and $60 \mathrm{~s}$ cases, respectively, while the pristine sample being $\sim 44 \mathrm{~cm}^{-1}$ (Supporting Information, Figure S2) [38]. Arial defect analysis was also performed using Raman mapping. Figure S3 (Supporting Information) presents the $I(\mathrm{D}) / I(2 \mathrm{D})$ intensity ratio mapping, which indicates the overall defects in the assynthesized graphene increase with acid treatment time from 30 to $60 \mathrm{~s}$. These results elucidate that the quality

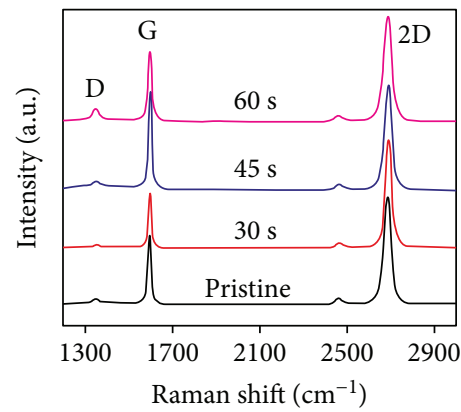

(a)

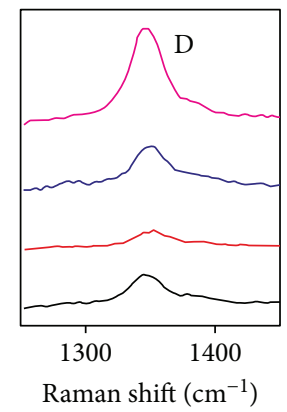

(b)
FIgURE 4: (a) Raman spectra of graphene films synthesized from different types of copper substrates. (b) Zoom-in regions of D peak from the whole Raman spectra. As shown in (b), the D peak intensity increases with prolonged acid treatment time.

of CVD graphene is closely related to the starting morphology of the growth substrate.

The effect of defect sites in graphene on electrical properties was investigated via conductivity and field-effect mobility measurements. The sheet resistance of graphene film prepared from the aforementioned conditions was measured by the 4-point probe as shown in Figure 1(a). Consistent with previous results, the $30 \mathrm{~s}$ sample again showed the lowest sheet resistance of $290 \pm 24 \Omega \cdot \mathrm{sq}^{-1}$, lower than that of the pristine graphene $\left(420 \pm 30 \Omega \cdot \mathrm{sq}^{-1}\right)$, potentially due to the low defects present on the graphene which act as the charge carrier scattering sites lowering the overall electrical properties in the graphene film [19]. The sheet resistance was also 
increased to $427 \pm 33$ and $643 \pm 45 \Omega \cdot \mathrm{sq}^{-1}$ with increasing acid treatment time ( 45 and $60 \mathrm{~s}$, resp.) and the associated increase in the degree of defect sites. We also note that large deviations of sheet resistance values also occur with increasing chemical treatments to the metal substrate. We suspect this phenomenon is closely related to the nonuniformity of copper surface morphology originating from the prolonged etching process.

The effect of surface morphology of graphene on the electrical property was further analyzed from the field-effect mobility measurement. Graphene-based field-effect transistor (FET) was fabricated by standard photolithography with $\mathrm{Ti}(10 \mathrm{~nm}) / \mathrm{Au}(50 \mathrm{~nm})$ contacts formed by thermal evaporation as illustrated in Figure 1(b). Consistent with the sheet resistance measurement, electron mobility was highest with $30 \mathrm{~s}$ sample $\left(1280 \mathrm{~cm}^{2} \cdot \mathrm{V}^{-1} \cdot \mathrm{s}^{-1}\right)$ and decreased to 957 and $613 \mathrm{~cm}^{2} \cdot \mathrm{V}^{-1} \cdot \mathrm{s}^{-1}$ for 45 and $60 \mathrm{~s}$ cases, respectively, with the pristine sample being $1156 \mathrm{~cm}^{2} \cdot \mathrm{V}^{-1} \cdot \mathrm{s}^{-1}$ (Figure $1(\mathrm{~b})$ ). The charge neutral point of graphene is slightly $p$-doped which is well-matched with Raman data shown in Figure S2.

\section{Conclusions}

In this work, we showed that the quality of CVD-grown graphene is closely related to the underlying surface morphology of the metal catalyst growth template. Via simple and facile modification of the metal catalyst with commonly used acid, high-quality graphene film could be readily obtained, an important factor to consider for the widespread industrial applications of graphene-based electronic devices. The acid reacts with the metal catalyst by etching the metal surface, thus removing the impurities present which tend to deteriorate the overall quality of graphene. We believe that the simple yet effective approach proposed in this study, which enables the synthesis of high-quality graphene films with low defects, will benefit a variety of graphene-based functional devices such as thin-film transistors, optoelectronic devices, and flexible electronics.

\section{Data Availability}

The SEM data used to support the findings of this study are included within the article. The Raman spectrum data used to support the findings of this study are included within the article. The OM image data used to support the findings of this study are included within the article. The FET mobility data used to support the findings of this study are included within the article. The transmittance data used to support the findings of this study are included within the supplementary information file(s). The Raman mapping data used to support the findings of this study are included within the supplementary information file(s).

\section{Conflicts of Interest}

The authors declare that they have no conflicts of interest.

\section{Acknowledgments}

This work was supported by Basic Science Research Program through the National Research Foundation of Korea (NRF) funded by the Ministry of Education (2015R1D1A1A0105791) and the Ministry of Science and ICT (2016R1A2B4014762).

\section{Supplementary Materials}

Figure S1: transmittance data shows the reduction of optical transmission by multilayer graphene under each condition. Figure S2: the FWHM value of the Raman $2 \mathrm{D}$ peak supports that the graphene quality depends on the nitric acid treatment time. Figure S3: Raman mapping results support that as-synthesized graphene shows high uniformity. (Supplementary Materials)

\section{References}

[1] K. S. Novoselov, A. K. Geim, S. V. Morozov et al., "Electric field effect in atomically thin carbon films," Science, vol. 306, no. 5696, pp. 666-669, 2004.

[2] A. K. Geim and K. S. Novoselov, "The rise of graphene," Nature Materials, vol. 6, no. 3, pp. 183-191, 2007.

[3] K. S. Novoselov, A. K. Geim, S. V. Morozov et al., "Twodimensional gas of massless Dirac fermions in graphene," Nature, vol. 438, no. 7065, pp. 197-200, 2005.

[4] Y. Zhang, Y. W. Tan, H. L. Stormer, and P. Kim, "Experimental observation of the quantum Hall effect and Berry's phase in graphene," Nature, vol. 438, no. 7065, pp. 201-204, 2005.

[5] T.-H. Han, Y. Lee, M. R. Choi et al., "Extremely efficient flexible organic light-emitting diodes with modified graphene anode," Nature Photonics, vol. 6, no. 2, pp. 105-110, 2012.

[6] A. K. Geim, "Graphene: status and prospects," Science, vol. 324, no. 5934, pp. 1530-1534, 2009.

[7] K. S. Novoselov, V. I. Fal'ko, L. Colombo, P. R. Gellert, M. G. Schwab, and K. Kim, "A roadmap for graphene," Nature, vol. 490, no. 7419, pp. 192-200, 2012.

[8] F. Xia, T. Mueller, Y. M. Lin, A. Valdes-Garcia, and P. Avouris, "Ultrafast graphene photodetector," Nature Nanotechnology, vol. 4, no. 12, pp. 839-843, 2009.

[9] H. Park, S. Chang, X. Zhou, J. Kong, T. Palacios, and S. Gradecak, "Flexible graphene electrode-based organic photovoltaics with record-high efficiency," Nano Letters, vol. 14, no. 9, pp. 5148-5154, 2014.

[10] R. Raccichini, A. Varzi, S. Passerini, and B. Scrosati, "The role of graphene for electrochemical energy storage," Nature Materials, vol. 14, no. 3, pp. 271-279, 2015.

[11] Y. Zhang, L. Zhang, and C. Zhou, "Review of chemical vapor deposition of graphene and related applications," Accounts of Chemical Research, vol. 46, no. 10, pp. 2329-2339, 2013.

[12] S. Stankovich, D. A. Dikin, R. D. Piner et al., "Synthesis of graphene-based nanosheets via chemical reduction of exfoliated graphite oxide," Carbon, vol. 45, no. 7, pp. 1558$1565,2007$.

[13] D. R. Dreyer, S. Park, C. W. Bielawski, and R. S. Ruoff, “The chemistry of graphene oxide," Chemical Society Reviews, vol. 39, no. 1, pp. 228-240, 2010. 
[14] X. Li, G. Zhang, X. Bai et al., "Highly conducting graphene sheets and Langmuir-Blodgett films," Nature Nanotechnology, vol. 3, no. 9, pp. 538-542, 2008.

[15] P. Blake, P. D. Brimicombe, R. R. Nair et al., "Graphene-based liquid crystal device," Nano Letters, vol. 8, no. 6, pp. 17041708, 2008.

[16] Y. Hernandez, V. Nicolosi, M. Lotya et al., "High-yield production of graphene by liquid-phase exfoliation of graphite," Nature Nanotechnology, vol. 3, no. 9, pp. 563-568, 2008.

[17] C. Berger, Z. Song, T. Li et al., "Ultrathin epitaxial graphite: $2 \mathrm{D}$ electron gas properties and a route toward graphenebased nanoelectronics," The Journal of Physical Chemistry B, vol. 108, no. 52, pp. 19912-19916, 2004.

[18] K. S. Kim, Y. Zhao, H. Jang et al., "Large-scale pattern growth of graphene films for stretchable transparent electrodes," Nature, vol. 457, no. 7230, pp. 706-710, 2009.

[19] O. V. Yazyev and Y. P. Chen, "Polycrystalline graphene and other two-dimensional materials," Nature Nanotechnology, vol. 9, no. 10, pp. 755-767, 2014.

[20] L. Gao, J. R. Guest, and N. P. Guisinger, "Epitaxial graphene on Cu(111)," Nano Letters, vol. 10, no. 9, pp. 3512-3516, 2010.

[21] Y. S. Dedkov, M. Fonin, U. Rudiger, and C. Laubschat, "Rashba effect in the graphene/Ni(111) system," Physical Review Letters, vol. 100, no. 10, pp. 107602-107604, 2008.

[22] J.-H. Lee, E. K. Lee, W. J. Joo et al., "Wafer-scale growth of single-crystal monolayer graphene on reusable hydrogenterminated germanium," Science, vol. 344, no. 6181, pp. 286289, 2014.

[23] Y. Hao, M. S. Bharathi, L. Wang et al., "The role of surface oxygen in the growth of large single-crystal graphene on copper," Science, vol. 342, no. 6159, pp. 720-723, 2013.

[24] S. Chen, H. Ji, H. Chou et al., "Millimeter-size single-crystal graphene by suppressing evaporative loss of $\mathrm{Cu}$ during low pressure chemical vapor deposition," Advanced Materials, vol. 25, no. 14, pp. 2062-2065, 2013.

[25] A. Mohsin, L. Liu, P. Liu et al., "Synthesis of millimetersize hexagon-shaped graphene single crystals on resolidified copper," ACS Nano, vol. 7, no. 10, pp. 8924-8931, 2013.

[26] S. M. Kim, A. Hsu, Y. H. Lee et al., "The effect of copper pre-cleaning on graphene synthesis," Nanotechnology, vol. 24, no. 36, pp. 365602-365608, 2013.

[27] M.-S. Kim, J.-M. Woo, D.-M. Geum, J. R. Rani, and J. H. Jang, "Effect of copper surface pre-treatment on the properties of CVD grown graphene," AIP Advances, vol. 4, no. 12, pp. 127107-127111, 2014.

[28] G. Zhao, X. Li, M. Huang et al., "The physics and chemistry of graphene-on-surfaces," Chemical Society Reviews, vol. 46, no. 15, pp. 4417-4449, 2017.

[29] A. Ibrahim, G. Nadhreen, S. Akhtar, F. M. Kafiah, and T. Laoui, "Study of the impact of chemical etching on $\mathrm{Cu}$ surface morphology, graphene growth and transfer on $\mathrm{SiO}_{2} /$ Si substrate," Carbon, vol. 123, pp. 402-414, 2017.

[30] A. T. Murdock, C. D. van Engers, J. Britton et al., "Targeted removal of copper foil surface impurities for improved synthesis of CVD graphene," Carbon, vol. 122, pp. 207-216, 2017.

[31] Y. H. Zhang, Z. Y. Chen, X. M. Ge et al., "A waterless cleaning method of the $\mathrm{Cu}$ foil for CVD graphene growth," Materials Letters, vol. 211, pp. 258-260, 2018.

[32] D. Senyildiz, O. T. Ogurtani, and G. Cambaz Buke, "The effects of acid pretreatment and surface stresses on the evolution of impurity clusters and graphene formation on Cu foil," Applied Surface Science, vol. 425, pp. 873-878, 2017.

[33] H. Wang, G. Wang, P. Bao et al., "Controllable synthesis of submillimeter single-crystal monolayer graphene domains on copper foils by suppressing nucleation," Journal of the American Chemical Society, vol. 134, no. 8, pp. 36273630, 2012.

[34] Y. Zhang, L. Gomez, F. N. Ishikawa et al., "Comparison of graphene growth on single-crystalline and polycrystalline $\mathrm{Ni}$ by chemical vapor deposition," The Journal of Physical Chemistry Letters, vol. 1, no. 20, pp. 3101-3107, 2010.

[35] A. Pirkle, J. Chan, A. Venugopal et al., "The effect of chemical residues on the physical and electrical properties of chemical vapor deposited graphene transferred to $\mathrm{SiO}_{2}$," Applied Physics Letters, vol. 99, no. 12, pp. 122108-122111, 2011.

[36] Z. Han, A. Kimouche, D. Kalita et al., "Homogeneous optical and electronic properties of graphene due to the suppression of multilayer patches during CVD on copper foils," Advanced Functional Materials, vol. 24, no. 7, pp. 964-970, 2014.

[37] A. Ismach, C. Druzgalski, S. Penwell et al., "Direct chemical vapor deposition of graphene on dielectric surfaces," Nano Letters, vol. 10, no. 5, pp. 1542-1548, 2010.

[38] S. Lee, K. Lee, and Z. Zhong, "Wafer scale homogeneous bilayer graphene films by chemical vapor deposition," Nano Letters, vol. 10, no. 11, pp. 4702-4707, 2010. 


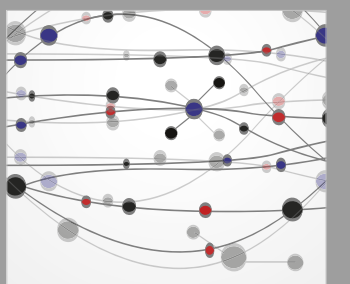

The Scientific World Journal
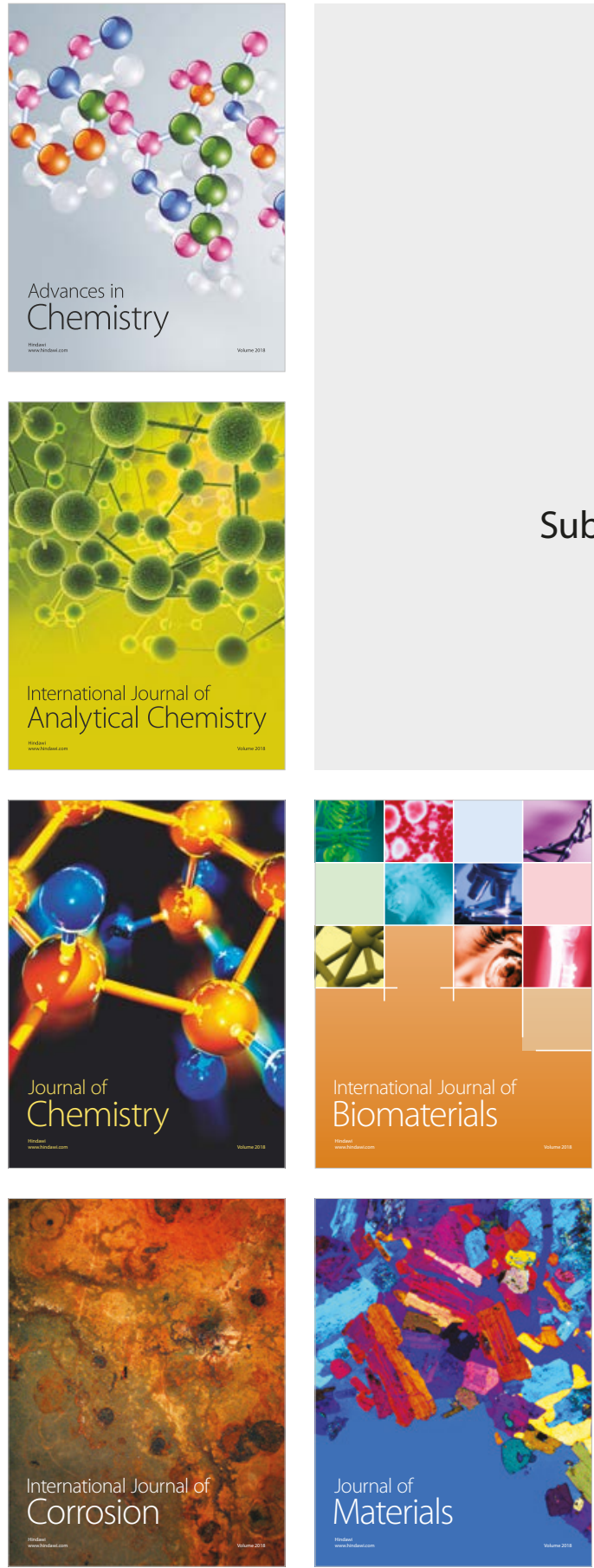

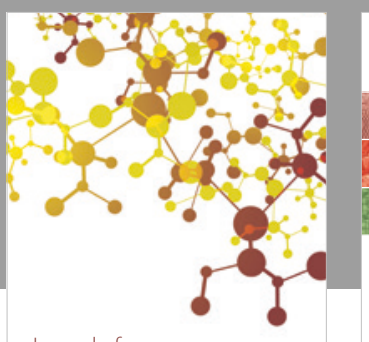

Journal of

Applied Chemistry
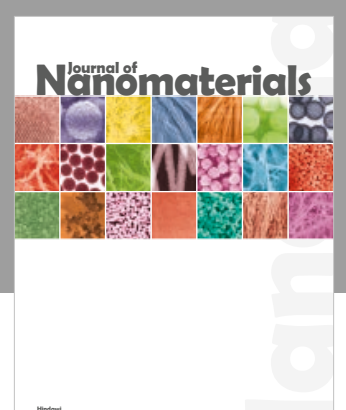

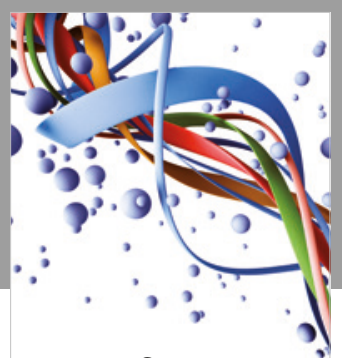

Scientifica

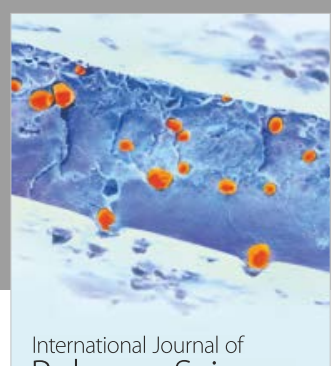

Polymer Science

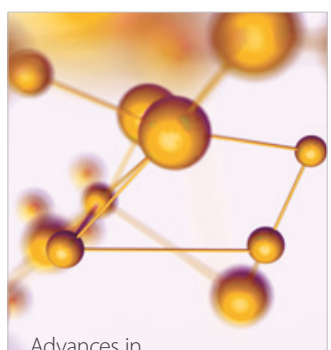

Physical Chemistry
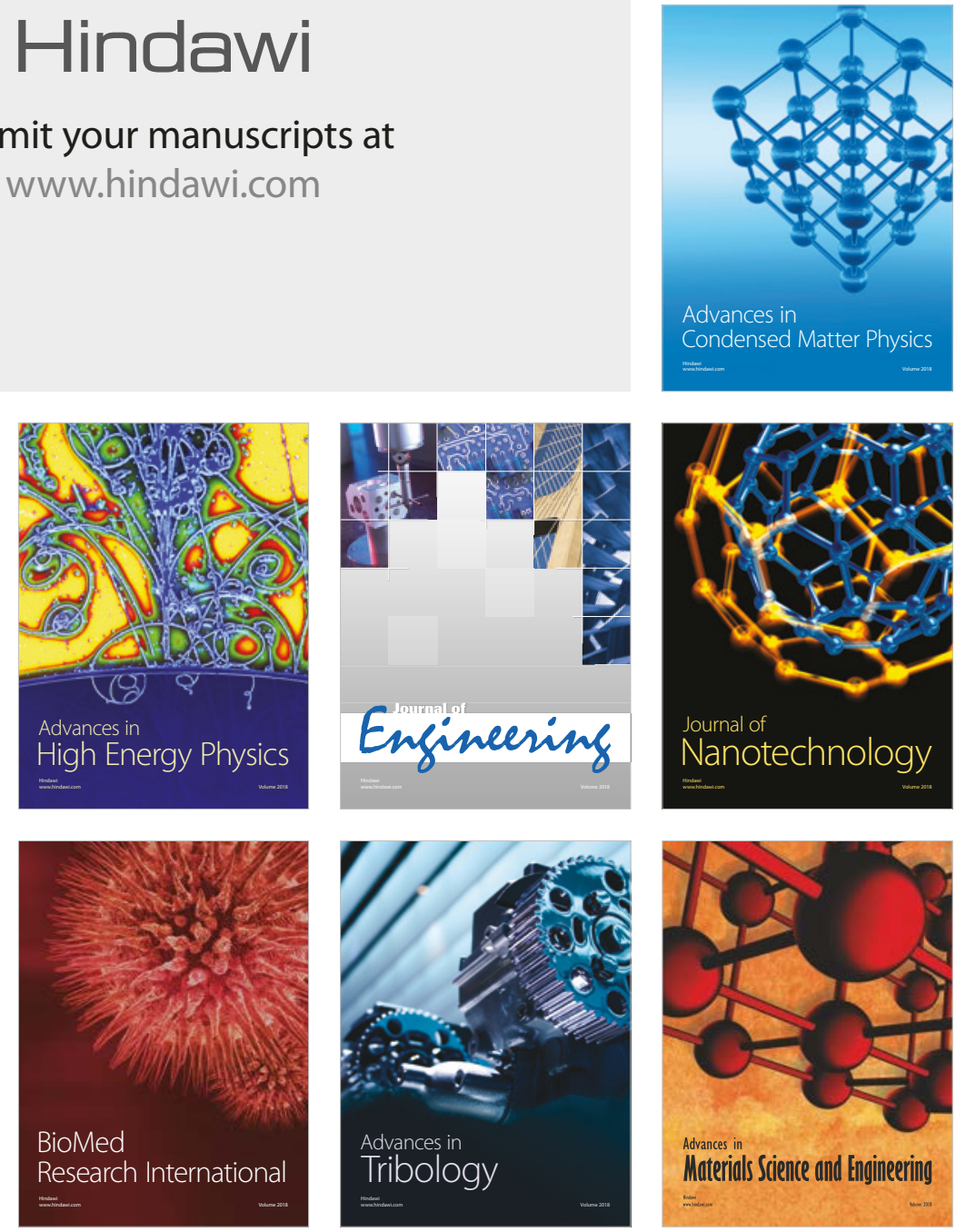